\title{
Wind Pressures on Structures by Proper Orthogonal
} Decomposition

\author{
Fanghui $\mathrm{Li}^{1}$, Ming $\mathrm{Gu}^{2}$, Zhenhua $\mathrm{Ni}^{3}$ and Shizhao Shen ${ }^{4}$ \\ 1. School of Architectural Engineering, Heilongjiang University, Harbin, China \\ 2. State Key Laboratory for Disaster Reduction in Civil Engineering, Tongji University, China \\ 3. Department of Civil Engineering, Shantou University, China \\ 4. School of Civil Engineering, Harbin Institute of Technology, China
}

\begin{abstract}
Proper orthogonal decomposition (POD) is an effective statistical technique for data reduction and feature extraction of the random field including the wind field. This paper introduces the theory of the POD and illustrates engineering of structures. Using the POD technique, it is shown that wind pressure data can be accurately reconstructed with a limited number of modes using the wind pressure data from wind tunnel test. Comparing the reconstructed values by POD with the original measured values from the wind tunnel test both in the time and frequency domains, it is concluded that the proper orthogonal decomposition(POD) is an efficient and practical technique for deriving the random wind pressure field from limited known data as shown in the pitched roof example in this paper.
\end{abstract}

Key words: Proper orthogonal decomposition, pitched roof, reconstruction of wind pressure field.

\section{Introduction}

Proper Orthogonal Decomposition (POD) is an effective method to analyze statistical characteristics of complex random fields. POD is a method of using a new coordinate system which represents the original random phenomina more efficiently, and greatly reduces the data storage [1-6]. POD technique represents a random process as a linear combination of the orthogonal eigenmodes and principal components, which are uncorrelated with each other. The POD technique has been applied in many fields including random variable, signal analysis, process identification, and many others [7-10]. This paper focuses on the applications of POD to wind engineering of structures.

The structures located in the boundary layer of atmospheric turbulence boundary layer usually have complex wind pressure fields because of the non-stationary turbulent flow, the flow separation and

Corresponding author: Fanghui $\mathrm{Li}, \mathrm{PhD}$, research field: wind engineering. E-mail: fhli_2000@163.com. reattachment. Pressure fluctuation is an important factor for the design of roofs because it is apt to cause numerous failures of structure claddings and roof covers. Based on the analysis of the eigenvalue problem of the covariance matrix or correlation matrix, POD applies an efficient technique to identify the wind pressure fields and adopts combination of simple series to describe the pressure fluctuation [11]. POD, as a space-time separation method, divides the pressure field into spatially varying parts, the eigenvectors of covariance matrix, which is independent of time, and time varying parts, the principal coordinates, which is independent of spatial position. The application of POD to wind engineering was first introduced to analyze pressure fluctuations on cooling tower by Armitt (1968) [12]. Best and Holmes (1983) investigated building wind loading using eigenvalues in the covariance integration method, pointed that the wind-induced RMS response of structures can be expressed as the response contributed by the first several eigenmodes [13]. Panofsky and Dutton (1984) 
proposed to simulate gust random samples by using principal coordinates [14]. Davenport applied POD to the research on wind velocity fields and wind pressure fields, provided an efficient method to generalization and simplification of wind load [15]. Bienkiewicz et al. analyzed the fluctuating wind pressure on the roof of a low-rise building. The mechanical essence of the lower eigenmodes was also discussed $[16,17]$.

In general, there are two different interpretations for the POD technique, the first interpretation as Karhunen-Loeve decomposition (KLD) and the second one considers that the POD consists of three methods: the KLD, the principal component analysis (PCA), and the singular value decomposition (SVD) [10].

In this paper, the derivation of POD is presented from the viewpoint of Rayleigh's quotient, which is independent of the original framework based on Mercer theorem, and the meanings of related variables are clarified. Then the application of POD to reconstruction of wind pressure field is introduced. Taking the pitched roof as an example, the reconstruction of wind pressure field was studied by using the wind pressure data measured in a wind tunnel test. The effectiveness and practicability of POD are illustrated by comparing the POD derived values with the original data in the time domain and frequency domain.

\section{POD Method}

$\{p(t)\}$ is the wind pressure vector measured simultaneously in wind tunnel model

$$
\{p(t)\}=\left\{p_{1}(t), p_{2}(t), \cdots p_{N}(t)\right\}^{T}
$$

Where, $p_{i}(t)=p\left(x_{i}, y_{i}, t\right)$ is the fluctuating pressure on the ith pressure tap.

An orthogonal coordinate is expected to be obtained, on which $\{p(t)\}$ has maximum projections. $\{\varphi\}_{n}$ is set as base vector of axis-n of the orthogonal coordinate. Accordingly the projection of pressure vector on the axis-n is:

$$
a_{n}(t)=\{p(t)\}^{T}\{\varphi\}_{n}=\{\varphi\}_{n}^{T}\{p(t)\}(n=1,2, \cdots, N)
$$

The formulation above is normalized:

$$
a_{n}(t)=\frac{\{\varphi\}_{n}^{T}\{p(t)\}}{\left(\{\varphi\}_{n}^{T}\{\varphi\}_{n}\right)^{1 / 2}}
$$

Where, $a_{n}(t)$ is principal coordinates. The following equation can be obtained by maximizing the projection using mean square method:

$$
\overline{a_{n}^{2}(t)}=\frac{\{\varphi\}_{n}^{T} \overline{\{p(t)\}\{p(t)\}^{T}}\{\varphi\}_{n}}{\{\varphi\}_{n}^{T}\{\varphi\}_{n}}=\frac{\{\varphi\}_{n}^{T}[c]\{\varphi\}_{n}}{\{\varphi\}_{n}^{T}\{\varphi\}_{n}}=\lambda_{n}
$$

$\{\varphi\}_{n}$ is derived from the equation above to obtain maximum value of $\lambda_{n}$, which is a kind of Rayleigh Quotient problem. Due to the characteristics of Rayleigh Quotient, it is known that the Rayleigh Quotient of Eq. (4) is stationary of eigenvector of covariance matrix $[c]$. Therefore, if and only if $\{\varphi\}_{n}$ satisfies the eigenvalue problem below, the $\overline{a_{n}^{2}(t)}$ is equal to stationary and the stationary, namely eigenvalue, is:

$$
[c]\{\varphi\}_{n}=\lambda_{n}\{\varphi\}_{n} \quad(n=1,2, \cdots, N)
$$

Assuming eigenmodes have been normalized, eigentmodes matrix and principal coordinates matrix are defined as follows:

$$
\begin{gathered}
{[\Phi]=\left[\{\varphi\}_{1},\{\varphi\}_{2}, \cdots,\{\varphi\}_{N}\right]} \\
\{a(t)\}=\left\{a_{1}(t), a_{2}(t), \cdots, a_{N}(t)\right\}^{T}
\end{gathered}
$$

Eq. (2) leads to:

$$
\{a(t)\}=[\Phi]^{T}\{p(t)\}
$$

According to the orthogonality of eigenmodes matrix $[\Phi]$, the following equation is given by Eq. (7)

$$
\{p(t)\}=[\Phi]\{a(t)\}=\sum_{n=1}^{N} a_{n}(t)\{\varphi\}_{n}
$$

POD divides the space and time-dependent pressure field into principal coordinates $a_{n}(t)$, which is independent of spatial position, and eigenmodes $\{\varphi\}_{n}$, which depends on spatial position.

The field-total sum of the mean square of the wind pressure field is equal to the sum of the eigenvalues, can be written

$$
\iint \overline{p^{2}(x, y, t)} d x d y=\sum_{n=1}^{N} \lambda_{n}
$$


The eigenvalue is the measure of the contribution of each eigenmode to the pressure mean squares.

In the following sections, the applications of POD to reconstruction of wind pressure field are investigated by using the wind pressure data which are measured simultaneously in a wind tunnel test.

\section{Wind Tunnel Test}

Wind tunnel test of the pitched roof building were conducted by the authors in January 2005 at the school of civil engineering Shantou University, using $1 / 60$ scale model. The $3 \mathrm{~m} \times 2 \mathrm{~m}$ cross-section model of the piched roof building is shown in Fig. 1. Its prototype has plan dimensions of $42 \mathrm{~m} \times 21 \mathrm{~m}$ with the roof pitch of $20^{\circ}$ and the eave height of $6 \mathrm{~m}$, in simulated boundary-layer turbulent flows, representing the open-country exposure with the power-law exponent of 0.16 that is in accordance with Chinese Loading Code of Building and Structures. The wind pressure data were measured simultaneously at numerous points across the roof surfaces using a multi-channel simultaneous fluctuating pressure measurement system. Four ZOC33 (manometric system module type) pressure modules were measured substantially simultaneously with 20480 samples and the sampling frequency of $312.5 \mathrm{~Hz}$ by using DSM3200 (electronic scanning valve pressure test system type) data acquisition system produced.

\section{Reconstruction of Pressure Fields}

Wind pressure fields on roof are difficult to be analyzed due to the large quantity of wind pressure

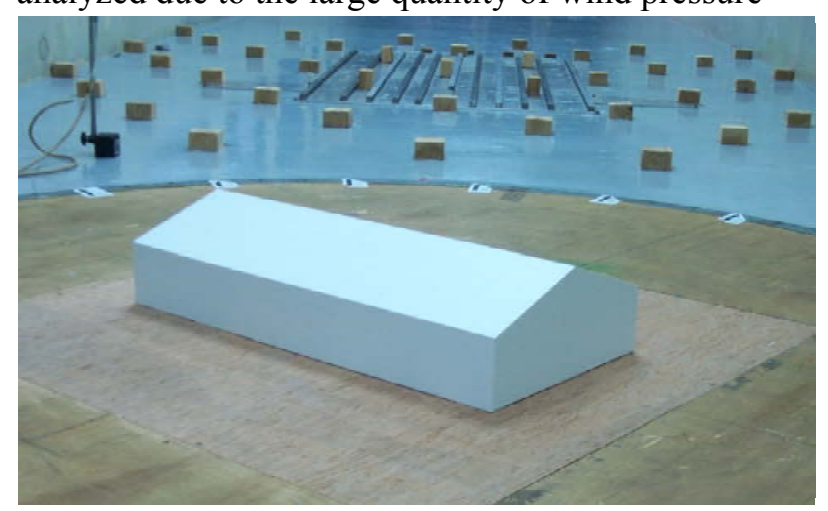

Fig. 1 Testing model in wind tunnel. data. A few of selected eigenmodes and principal coordinates are utilized to reconstruct pressure field and extract the properties of random pressure fields by POD. The wind pressure coefficient at a point $(\mathrm{x}, \mathrm{y})$ on the roof surface can be expressed as

$$
C_{p}(x, y, t)=\sum_{m=1}^{M} a_{m}(t) \varphi_{m}(x, y)
$$

where $\mathrm{M}$ is the number of eigenmodes to be adopted, $a_{m}(t)$ is the principle coordinates, $\varphi_{m}(x, y)$ is the eigenmodes. The number of modes that need to be considered for exact reconstruction, namely, $\mathrm{M}$ is smaller than $\mathrm{N}$. The original wind pressure field may be approximated by Eq. (10), this is so-called the reconstruction of wind pressure field.

Combining pressure data which were measured simultaneously in wind tunnel, the wind pressure field on the pitched roof model is reconstructed by POD. The distribution of pressure taps is shown in Fig. 2. Table 1 gives the comparison of contributions from the first ten modal energies and associated accumulated energies calculated from the eigenvalue problem with or without the mean pressure components. It was found that the modal energy with mean wind pressure components included is much larger than the same modal energy without mean pressure values. The analysis of eigenvalue problem demonstrates that the 1 st eigenvalue of correlation matrix contributes significantly. Therefore, reconstruction by the 1 st eigenmode is usually accurate enough for most of pressure taps. Table 2 shows the mean wind pressure value on the typical taps of the pitched roof. The closed comparison between reconstructed and real mean wind pressure coefficients at typical pressure taps validates the efficiency of the proposed reconstruction method.

Fig. 3 shows the comparison between original (solid line) and reconstructed (dotted line) pressure time series and auto-spectra of tap 1, in which the notation "M modes"means $\mathrm{M}$ modes were adopted in pressure reconstruction based on POD technique. It is indicated that the errors of both time and frequency domain analysis are reduced as the number of adopted modes 


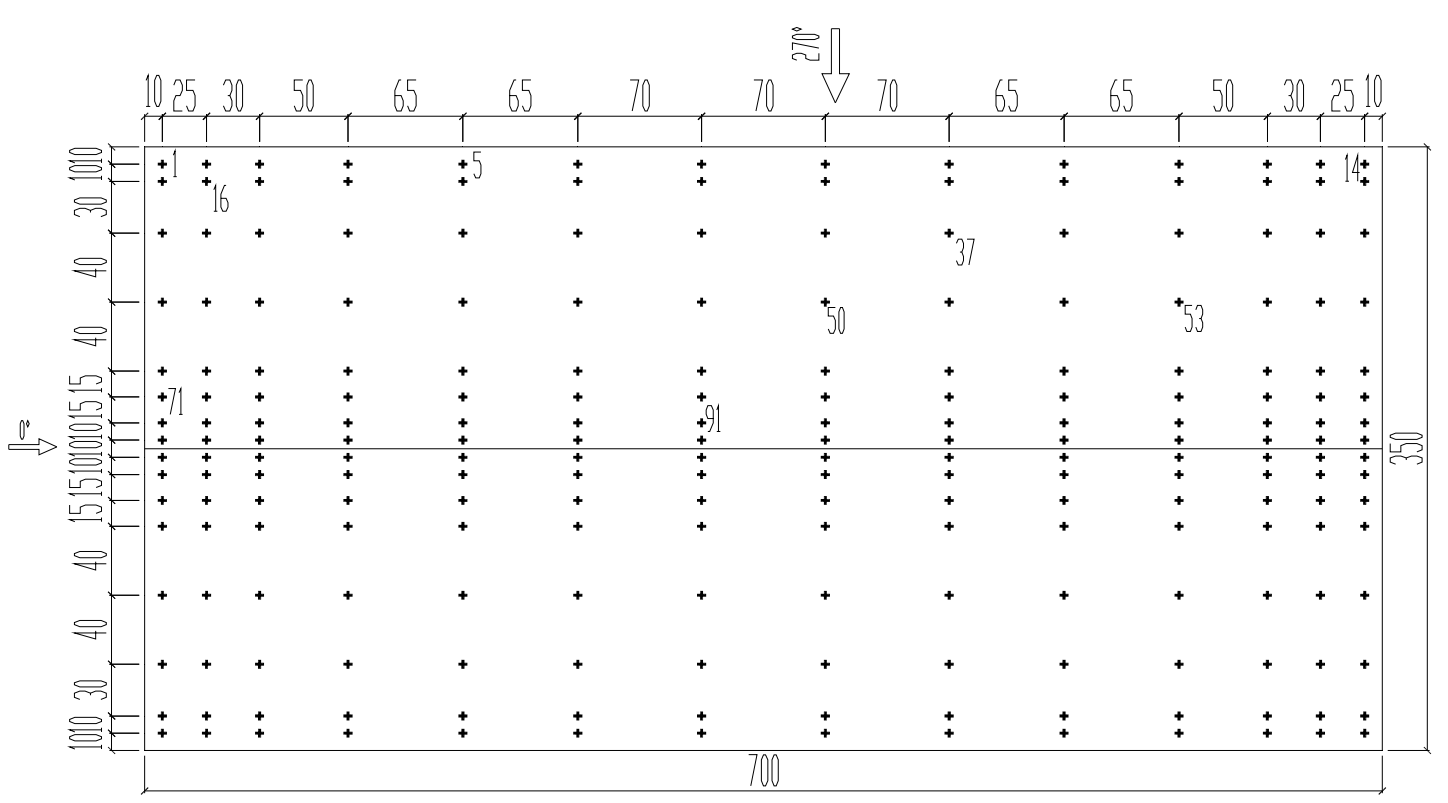

Fig. 2 Arrangement of pressure taps on roof (unit: $\mathbf{m m}$ ).

Table 1 Comparison of the first 10 modal energies and associated accumulated energies.

\begin{tabular}{c|c|c|c|c}
\hline \multirow{2}{*}{ Mode } & \multicolumn{2}{|c|}{ Mean Included } & \multicolumn{2}{c}{ Mean Excluded } \\
\cline { 2 - 5 } & Modal Energy (\%) & Accumulated Energy (\%) & Modal Energy (\%) & Accumulated Energy (\%) \\
\hline 1 & 86.46 & 86.46 & 16.23 & 16.23 \\
\hline 2 & 1.13 & 87.59 & 7.01 & 23.24 \\
\hline 3 & 0.91 & 88.50 & 5.68 & 28.92 \\
\hline 4 & 0.63 & 89.12 & 3.52 & 32.44 \\
\hline 5 & 0.56 & 89.68 & 3.40 & 35.84 \\
\hline 6 & 0.51 & 90.19 & 3.15 & 38.99 \\
\hline 7 & 0.47 & 90.66 & 2.95 & 41.94 \\
\hline 8 & 0.45 & 91.11 & 2.79 & 44.72 \\
\hline 9 & 0.40 & 91.51 & 2.49 & 47.22 \\
\hline 10 & 0.32 & 91.83 & 1.96 & 49.18 \\
\hline
\end{tabular}

Table 2 Comparison between reconstructed and real mean wind pressure coefficient at typical pressure taps.

\begin{tabular}{cccc}
\hline $\begin{array}{c}\text { Number of } \\
\text { taps }\end{array}$ & $\begin{array}{c}\text { Reconstructed } \\
\text { value }\end{array}$ & $\begin{array}{c}\text { Real } \\
\text { value }\end{array}$ & Error(\%) \\
\hline 1 & -0.5644 & -0.5644 & 0.00 \\
\hline 5 & -0.1003 & -0.1005 & -0.23 \\
\hline 53 & -0.0336 & -0.0331 & 1.48 \\
\hline 71 & -0.4792 & -0.4785 & 0.15 \\
\hline
\end{tabular}

are increased. If the number of adopted modes is large enough, the wind pressure field can be reconstructed exactly with this method. The efficiency of reconstruction depends on the location of reconstructed pressure taps. The reconstruction of regions with stronger wind pressure is more efficient. The errors are mainly shown in high-frequency area when the modes adopted are deficient.

\section{Conclusions}

Proper orthogonal decomposition (POD) is a useful and efficient statistical technique for description of random fields. It has gained widespread application in wind engineering of spatial structures. In this paper, the POD was introduced in the form of Rayleigh's quotient. The validity and efficiency of the POD in the wind 

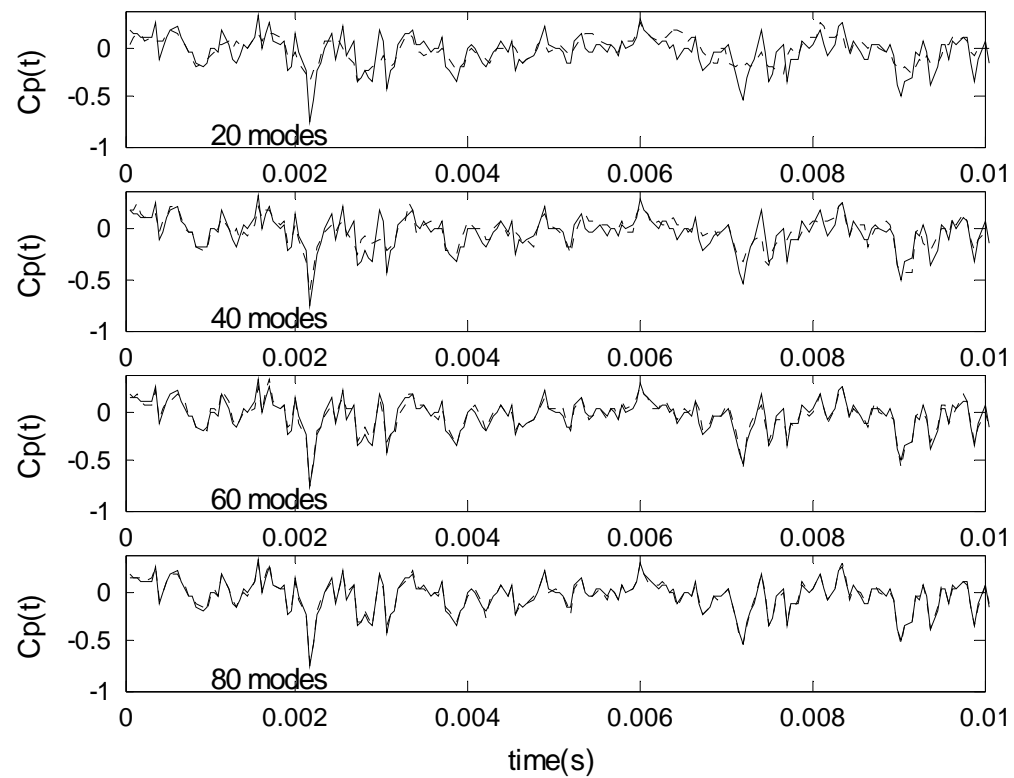

(a) wind pressure comparison
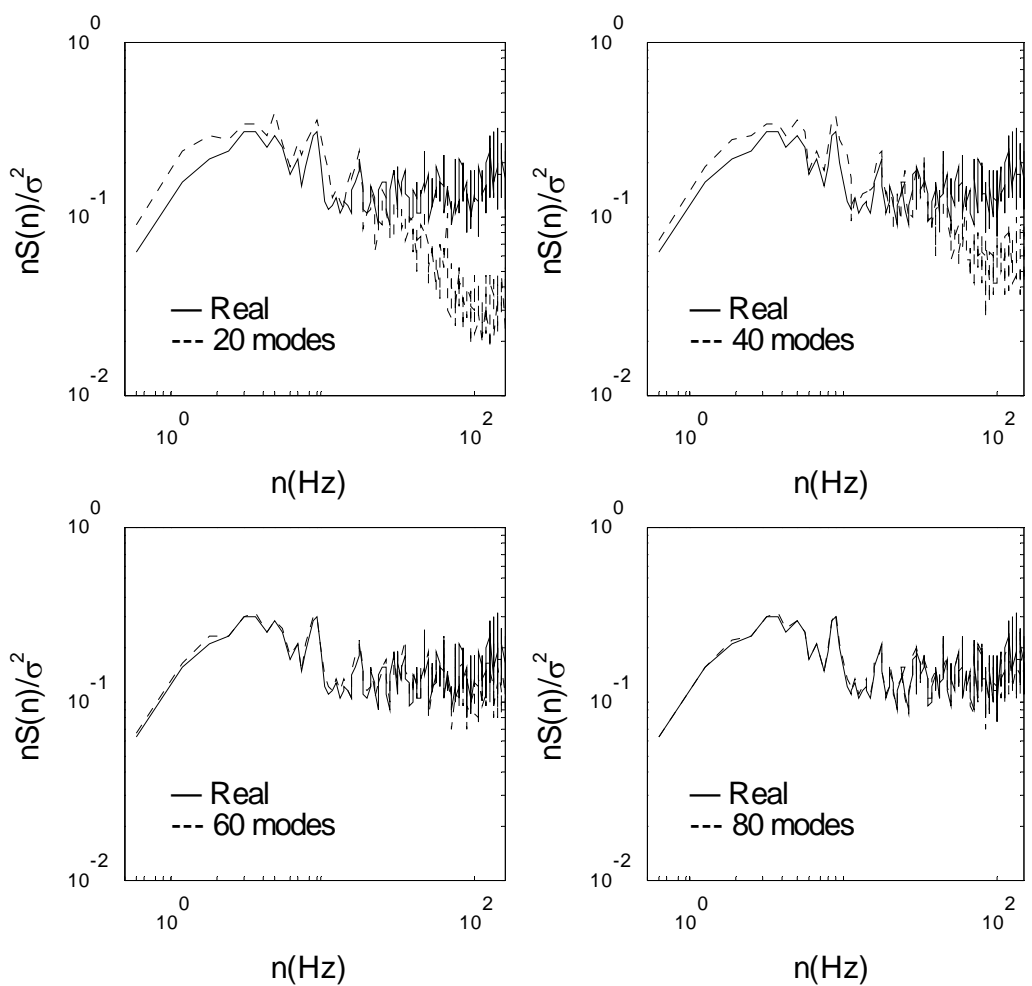

(b) auto-spectra comparison

Fig. 3 Comparison between original (solid line) and reconstructed (dotted line) pressure time series and auto-spectra of tap 1.

engineering application was demonstrated using a pitched roof example where the values from the simultaneously measured wind pressure coefficients are compared with the POD reconstructed values using limited number of eigenmodes and principal coordinates. It was found that the values are closed to almost identical, and that the first eigenmode contributes significantly to the results. In this paper, the POD theory is presented, the reconstruction of a long-span pitched roof is performed. 
(1) The derivation of POD is presented from the viewpoint of Rayleigh's quotient, which is independent of the original theory framework which is based on mathematics, and the relationship between eigenmodes and principal coordinates are clarified with unambiguous mechanical meanings.

(2) The limited number of eigenmodes and principal coordinates is accurate enough for reconstruction of wind fields on pitched roof. The efficiency of the proposed method is illustrated by comparing with original data in the time domain and frequency domain. Moreover, the description of wind field can be simplified making use of series combination of eigenmodes and principal coordinates.

\section{Acknowledgements}

The authors are grateful for the support of this research by the Committee of National Science Foundation of China (50908077) and Foundation of Heilongjiang Province Educational Committee (11551368).

\section{References}

[1] X. Gillamd, D. Smith and F. Wu, Proper orthogonal decomposition modeling for full-scale pressure fields, 14th Engineering Mechanics Conference, Austin Texas (2000) 21-24.

[2] C. Wolter, M. A. Trindade and R. Sampaio, Obtaining mode shapes through the karhunen-loeve expansion for distributed-parameter linear systems, Shock and Vibration 9 (2002) 177-192.

[3] G. Kerschen, B. F. Feeny and J. C. Golinval, On the exploitation of chaos to build reduced-order models, Comput.Methods Appl. Mech. Engrg 192 (2003) 1785-1795.

[4] P. G. Cizmas, A. Palacios, T. O. Brien, Proper orthogonal decomposition of spatio-temporal patterns in fluidized beds, Chemical Engineering Science 58 (2003) 4417-4427.

[5] B. Bienkiewicz, New tools in wind engineering, J. Wind Eng. Ind. Aerodyn 65 (1996) 279-300.

[6] Y. Tamura, S. Suganuma and H. Kikuchi, Proper orthogonal decomposition of random wind pressure field, Journal of Fluids and Structures 13 (1999) 1069-1095.

[7] P. Hemon and F. Santi, Applications of biorthogonal decompositions in fluid-structure interactions, Journal of Fluids and Structures 17 (2003) 1123-1143.

[8] J. C. Slater, C. L. Pettit and P. S. BERAN, In-situ residual tracking in reduced order modeling, Shock and Vibration 9 (2002) 105-121.

[9] J. D. Holmes, Analysis and synthesis of pressure fluctuations on bluff bodies using eigenvectors, J. Wind Eng. Ind. Aerodyn 33 (1990) 219-230.

[10] C. G. Wu, Y. C. Liang and W. Z. Lin, A note on equivalence of proper orthogonal decomposition methods, Journal of Sound and Vibration 265 (2003) 1103-1110.

[11] A. Kareem, Analysis and moelling of wind effects: numerical techniques, Wind Engineering into the 21st Century, Rotterdam, 1999.

[12] J. Armitt, Eigenvector analysis of pressure fluctuations on the West Burton instrumented cooling tower, Internal Report RD/L/N 114/68, Central Electricity Research Laboratories (UK), 1968.

[13] R. J. Best and J. D. Holmes, Use of eigenvalues in the covariance integration method for determination of wind load effects, J. Wind Eng. Ind. Aerodyn 13 (1983) 359-370.

[14] S. H. Jeong, Simulation of large wind pressures by gusts on a bluff structure, Wind and Structures 7 (5) (2004) 333-344.

[15] A. G. Davenport, How can we simplify and generalize wind loads? J. Wind Eng. Ind. Aerodyn 54/55 (1995) 657-669.

[16] B. Bienkiewicz, Y. Tamura, H. J. Ham, H. Ueda and K. Hibi, Proper orthogonal decomposition and reconstruction of multi-channel roof pressure, J. Wind Eng. Ind. Aerodyn 54/55 (1995) 369-381.

[17] B. Bienkiewicz, H. J. Ham and Y. Sun, Proper orthogonal decomposition and reconstruction of roof pressure, J. Wind Eng. Ind. Aerodyn 50 (1993) 193-202. 